\title{
Neutron-multiwave-interference experiments with many resonance coils
}

\author{
S. V. Grigoriev, ${ }^{1,2, *}$ Yu. O. Chetverikov, ${ }^{1}$ A. V. Syromyatnikov, ${ }^{1}$ W. H. Kraan, ${ }^{2}$ and M. Th. Rekveldt ${ }^{2}$ \\ ${ }^{1}$ Petersburg Nuclear Physics Institute, Gatchina, St. Petersburg 188350, Russia \\ ${ }^{2}$ Interfacultair Reactor Instituut, TU-Delft, $2629 \mathrm{JB}$ Delft, The Netherlands
}

(Received 29 April 2003; published 12 September 2003)

\begin{abstract}
Neutron-multiwave-interference phenomena based on Ramsey's resonance method of "separated oscillating fields" are studied. A neutron passes through $N$ successive resonant coils $\left(\hbar \omega_{0}=2 \mu_{n} B_{0}\right)$, which flip the neutron spin with a probability $\rho$ smaller than 1 . These coils are separated by path lengths $L$ over which a homogeneous field $B_{1}$ is present. Because the spin-flip probability $\rho$ is smaller than 1 , the number of waves for a neutron is doubled after each flipper, so as to produce $2^{N}$ neutron waves at the end of the setup. The phase difference between any pair of waves is a multiple of a "phase quantum" determined by the line integral of the field difference $B_{1}-B_{0}$ over the length $L$. Highly regular patterns of the quantum-mechanical probability $R$ in $\left(B_{1}, \rho\right)$ space appear due to pair interference between individual waves. Possible applications of this phenomenon, such as a direct measurement of $n$-particle correlation function, are pointed out.
\end{abstract}

DOI: $10.1103 /$ PhysRevA.68.033603

PACS number(s): 03.75.Dg, 42.87.Bg

\section{INTRODUCTION}

Multipath interference in optics and multimode interference in dynamical systems has recently emerged as an extremely active field of research. A coherently illuminated diffraction grating produces remarkably rich series of selfimages in a plane beyond the grating. This effect has recently received much attention as a fundamental optical phenomenon $[1,2]$. It appears that this phenomenon based on the multipath interference of light has much similarity with multimode interference in the evolution of the wave packet in a system described by quantum mechanics [3]. Such a system with a broad spectrum of excitations, when all the levels are populated, reveals rich interference patterns both in time and in space $[4,5]$. Particularly, the large scale interference leads to the well-ordered long-range regularities (such as quantum revivals [5]) in time-space probability distribution of the wave function. When a Gaussian wave packet propagates in time and space, a regular structure emerges for the probability density, the so-called quantum carpet, which becomes an object of study.

Therefore it is of great interest to prepare a wave packet in a controlled way and measure its multimode or multipath interference. In this paper we use Ramsey's resonance method of the separated oscillating fields $[6,7]$ to study neutron multipath wave interference. In his works Ramsey considered a beam with spin $\pm \frac{1}{2}$ particles (a two level system of which a neutron is a good example) passing through a couple of static combined with oscillating fields in resonance with the particle's magnetic moment, separated by homogeneous or zero magnetic field. He calculated the transition probability between the levels present in this field configuration. The calculations demonstrated that neutron wave interference occurs between two possible wave paths through spinmomentum space. It was discussed in Ref. [7] that this method of producing interference between neutron waves along the different paths in momentum space has analogy

\footnotetext{
*Email address: grigor@pnpi.spb.ru
}

with the interference produced along different paths in real space, i.e., with two-slit experiment. Recently, the interference of two neutron waves having different prehistories in spin-momentum space was experimentally observed $[8,9]$. It should be noted that this phenomenon has much in common with the neutron resonant spin echo method recently developed [10-12], which is based on the earlier works on the resonant interaction of neutrons with time-dependent magnetic fields [13-15].

In this paper we study the probability density of the neutron passed through $N$ resonant coils flipping the neutron spin with a probability $\rho$ between 0 and 1 and separated one from another by a homogeneous field $B_{1}$ of length $L$. The same configuration was described in Ref. [6,7] for two coils only. When the neutron with spin parallel to the quantization axis enters the first resonant coil the neutron wave is split into two, with different spin states. In the subsequent area with magnetic field $B_{1}$, these two neutron waves experience the magnetic field differently because of their different spin states, i.e., each wave collects a different (opposite) phase shift. The next resonance coil produces a new splitting of each of the two neutron waves, thus making four waves. Hence the full device consisting of $N$ resonance coils produces $2^{N}$ neutron waves. These waves interfere and each pair of them contributes to a highly regular pattern of quantummechanical probability in a two-dimensional space subtended by the "axes" of spin-flip probability $\rho$ and line integral $\left(B_{1}-B_{0}\right) L$.

We give a theoretical treatment of this problem. The formulas describing the appearance of $2 N$ neutron waves in this many-resonance experiment are derived in Sec. II. The numerical calculations for large numbers of the resonance coils are given in Sec. III. Section IV gives details concerning the experiment for system of six resonance coils. Section V presents both short discussion and final conclusion.

\section{NEUTRON WAVE INTERFERENCE IN RAMSEY'S RESONANCE METHOD}

\section{A. Case of two resonant coils}

The simplest way to understand interference between neutron waves in Ramsey's resonance method of the separated 

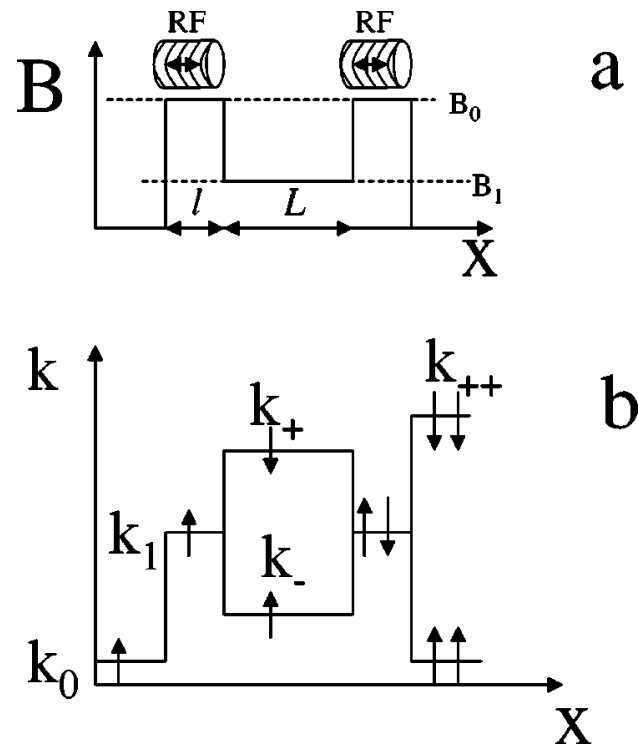

FIG. 1. (a) Sketch of a system of two radio frequency (RF) coils inside static field $B_{0}$ of length $l$ acting as RF resonant spin flippers with a field $B_{1}$ of length $L$ between them. (b) $(k, x)$ diagram of the wave vectors as a function of a position along the beam.

oscillating fields is to use a quantum-mechanical approach to the problem [7-12]. Let us consider a plane neutron wave with initial wave number $k_{0}$, energy $\hbar \omega$, and one spin state $\left(\frac{1}{0}\right)$. So the wave is represented as $\exp \left(i k_{0} x\right) \exp (-i \omega t)$. This wave travels along the $x$ axis through the configuration of the magnetic fields shown in Fig. 1(a). As soon as the neutron enters the field $B_{0}$, the wave number $k_{0}$ changes to $k_{1}$. Due to the energy conservation law, the total energy $\hbar \omega$ does not change and the resulting change in wave number satisfies the equation $\hbar^{2} k_{0}^{2} /\left(2 m_{n}\right)=\hbar^{2} k_{1}^{2} /\left(2 m_{n}\right)-\mu_{n} B_{0}$. Hence $k_{1}$ and $k_{0}$ differ in first approximation as:

$$
k_{1}=k_{0}+\mu_{n} B_{0} /(\hbar v),
$$

where $m_{n}, \mu_{n}$, and $v$ are the mass, the magnetic moment, and the velocity of the neutron, respectively.

Along the path length where the field equals $B_{0}$, an oscillating, field with frequency $\omega_{0}$ [radio frequency (RF)], perpendicular to $B_{0}$, is generated by a "resonant" coil. Its frequency is adjusted such that the photon energy exactly equals the Zeeman energy difference between the two spin eigenstates of the neutron in the static field:

$$
\hbar \omega_{0}=-2 \mu_{n} B_{0} .
$$

By means of the strength $B_{R F}$ of the RF field, the probability $\rho$ for the neutron to change its spin eigenstates can be changed between 0 and 1 . If spin flipping occurs, the total energy of the neutrons is not conserved because a photon of energy $\hbar \omega_{0}$ is exchanged between the neutron state and the RF field. Then, the neutron spin state with momentum $k_{1}$ will gain or lose an amount of potential energy $\Delta E$ $=2 \mu_{n} B_{0}$. When the neutron leaves the field $B_{0}$ and enters the path length with field $B_{1}$, its potential energy is released as a kinetic-energy change. Since we consider not complete but a partial spin flip, at this point the neutron wave is split into two plane waves with wave number $k_{+}$with the corresponding spin state $\left(\frac{0}{1}\right)$ (down) and wave number $k_{-}$with the spin state $\left(\frac{1}{0}\right)$ (up). Again, due to the energy conservation law, the total energy of each of these two waves does not change at the transition from $B_{0}$ to $B_{1}$, so their wave numbers $k^{+}$and $k^{-}$satisfy

$$
\frac{\hbar^{2} k_{1}^{2}}{2 m_{n}}=\frac{\hbar^{2} k_{+}^{2}}{2 m_{n}}-\mu_{n}\left(B_{0}-B_{1}\right)
$$

and

$$
\frac{\hbar^{2} k_{1}^{2}}{2 m_{n}}=\frac{\hbar^{2} k_{-}^{2}}{2 m_{n}}+\mu_{n}\left(B_{0}-B_{1}\right),
$$

from which one finds $k_{+}$and $k_{-}$in first approximation:

$$
k_{ \pm}=k_{1} \pm \frac{\mu_{n} \Delta B}{\hbar v}=k_{0}+\frac{\mu_{n}\left(B_{0} \pm \Delta B\right)}{\hbar v},
$$

where $\Delta B=B_{0}-B_{1}$.

Thus, after the resonance coil with partial spin flip, the initial neutron wave is split into a "nonflipped" and a "flipped" part with wave vectors $k_{-}$and $k_{+}$and with corresponding energies $\hbar \omega$ and $\hbar\left(\omega+\omega_{0}\right)$, respectively. In the subsequent region with static field $B_{1}$, these waves interfere and their phase difference $\phi(x, t)=\int_{0}^{x}\left[k_{+}\left(x^{\prime}\right)\right.$ $\left.-k_{-}\left(x^{\prime}\right)\right] d x^{\prime}-\omega_{0} t$ implies an effective precession in space. This spatial precession may take place even in zero field, i.e., when $B_{1}=0 \quad$ [so-called "zero-field (ZF) precession"] $[11,12]$. However, in a static experiment this is unobservable because the phase difference between the two interfering waves continues to grow in time at the rate $\omega_{0}$. Nevertheless, such a non-stationary interference pattern exists and was observed for the first time by Badurek et al. [15] using stroboscopic neutron detection.

This time-dependent behavior may be halted by transmitting the neutron through the next resonance coil, identical to the first one and placed in a static field equal to $B_{0}$. Upon entering the field $B_{0}, k_{+}$for the spin-down state and $k_{-}$for the spin-up state both return to $k_{1}$. In this second resonance coil the wave with spin down (i.e., flipped in the first coil) can only emit a photon, thus loosing the amount of the energy $\hbar \omega_{0}$. On the other hand, the wave with spin up (i.e., nonflipped in the first coil) can only absorb a photon, thus absorbing a quantum $\hbar \omega_{0}$. Since we suppose the spin flip in the second resonance coil again to be partial, both waves split again into two new waves. Upon leaving the second path length with the static field $B_{0}$ the potential energy of all waves is released as a kinetic-energy change. The waves with spin state up change their wave number $k_{1}$ into $k_{0}$; the waves with spin state down from $k_{1}$ into $k_{++}=k_{0}$ $+2 m_{n} B_{0} /(\hbar v)$. There are two waves at the upper energy level (each absorbed a photon in the first or the second coil) and two waves at the lower energy level (one absorbed a photon in the first coil and emitted it in the second; the other neither emitted nor absorbed a photon). The energies of each 
pair are finally equal. Thus, the growth of both the spatial and time phase differences for the two pairs of waves (separated in $\omega-k$ space) is halted.

Figure 1(b) is the $(k, x)$ diagram, i.e., diagram of two different wave-vector paths through the system of the magnetic fields [Fig. 1(a)] as a function of position along the beam. The phase shift between pairs of neutron waves after leaving the second coil equals $\Delta \phi=\int\left[k_{+}(x)-k_{-}(x)\right] d x$, where the integral is taken over the whole length of the two coil systems and is proportional to the area between two $k$ levels sketched in Fig. 1(b). As noted earlier, the interference appears only when $0<\rho<1$, so two $k$ levels in the space between flippers will be simultaneously occupied by a neutron.

The splitting of the waves is fully connected to the spin part of the wave function. One can follow in Fig. 1(b) what happens to both spin states along the beam path. It is indicated at different positions in Fig. 1 by the arrows $\uparrow$ and $\downarrow$, which correspond to the spinor components $\uparrow=\left(\frac{1}{0}\right)$ and $\downarrow$ $=\left(\frac{0}{1}\right)$. Each $k$ level can be identified by one spinor component only. Coefficients in the spinors accounting for the spin state of the initial wave and depending on the spin-flip probability of the RF coils determine the occupation numbers of the neutron wave on each level, i.e., along each wave-vector path in the diagram.

\section{B. Case of many resonant coils}

Let us assume a plane neutron wave travelling along the $x$ axis through the configuration of magnetic fields $B_{0}$ and $B_{1}$, which is $N$ times repeated, as shown in Fig. 2. RF coils in the path length with field $B_{0}$ are operated at the resonance frequency. Let us suppose for simplicity that all resonance coils (the so-called flippers) operate with the flipping probability $\rho=1 / 2$. The case of arbitrary $\rho$ will be treated in the following section.

Therefore, upon leaving the coil and entering the field $B_{1}$, the neutron wave is split into two with wave numbers $k_{+}$and $k_{-}$. Then, after the second coil each of these waves is split again into two equally populated waves and so on. Thus, after $N$ resonance coils the initial wave is split into two groups of $2^{N-1}$ neutron waves with small amplitudes of $(1 / 2)^{N}$ of the initial wave. Half of them now have energy $\hbar\left(\omega+\omega_{0}\right)$, they were flipped an odd number of times and therefore they have the spin state down. The other half have the energy $\hbar \omega$, as the initial wave had. They were flipped an even number of times or one of them was not flipped at all. Therefore they have the "up" spin state. The first group of the waves is located at the upper $k$ level of diagram [Fig. 2(b)] with its own energy and spin state; the second group is at the lower $k$ level with another energy and opposite spin state.

In fact, the neutron waves inside each group differ only in phase, since each of them has its own unique path in $(k-x)$ diagram. It is convenient to follow the relative phase shifts $\Delta \varphi$ of the individual wave with respect to the phase value $\varphi_{0}=k_{1} x$ [Fig. 2(c)]. The number of waves with an equal phase shift $\Delta \varphi$ obeys the simple binomial distribution. In the space after the system of $N$ resonance coils many pairs of

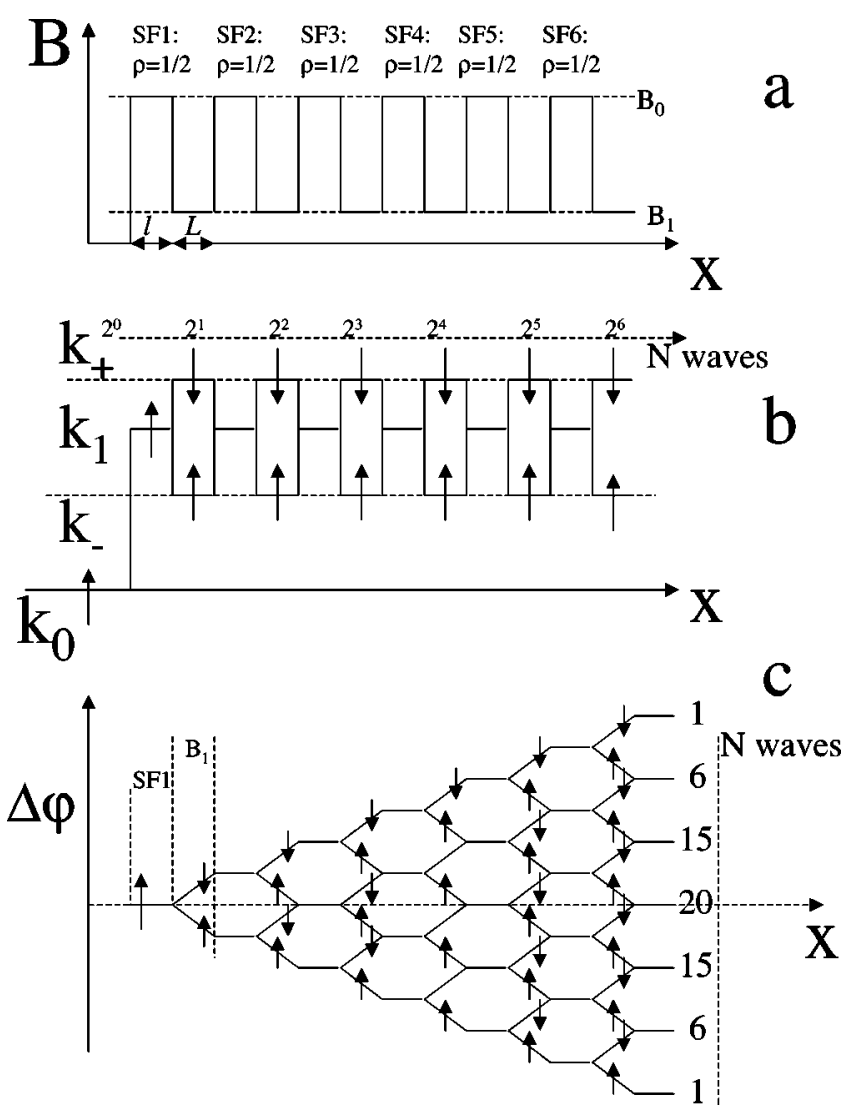

FIG. 2. (a) Sketch of the system with many resonant coils in field $B_{0}$ separated by segments with field $B_{1}$. (b) $(k, x)$ diagram of the wave vectors as a function of a position along the beam. (c) Diagram of the phase $\Delta \varphi$ of the wave produced in successive resonance coils along the beam relative to a wave which would go at undisturbed level $k=k_{1}$ through the system.

waves interfere and the phase difference for an arbitrary pair $\phi=m \Delta \phi$, where $m=0,1, \ldots N$, and

$$
\Delta \phi=\int_{0}^{l}\left[k_{+}\left(x^{\prime}\right)-k_{-}\left(x^{\prime}\right)\right] d x^{\prime}
$$

is the integral over one path section with field $B_{1}$. Thus, $\Delta \phi$ is a quantum of phase. Each pair of the waves contributes to the interference pattern downstream the system. What is this multiwave interference pattern? What kind of the rules does it obey? How will this pattern change if the spin-flip probability of the coil will differ from $1 / 2$ and therefore the amplitudes of the waves will not be as simple as $(1 / 2)^{N}$ ? These and some more questions will be answered in the following sections.

\section{Quantitative approach}

In order to describe quantitatively what happens with the initial plane neutron wave, we have to treat its behavior as a solution of the Schrödinger equation. We do so in the way as was already done for one resonance coil [6-8]. Thus we consider the wave of a neutron with velocity $v$ passing through the first resonance coil of length $l$ producing a trans- 
verse rotating magnetic field with frequency $\omega_{0}$ and amplitude $B_{R F}$ and inside a static field $B_{0}$. Its spin state is written as $\alpha(t)\left(\frac{1}{0}\right)+\beta(t)\left(\frac{0}{1}\right)$, where the coefficients $\alpha$ and $\beta$ satisfy $\alpha^{2}+\beta^{2}=1$ at any time. The Schrödinger equation of the system can be written as

$$
i \hbar \frac{d \Psi}{d t}=\left(\begin{array}{cc}
-\mu_{n} B_{0} & \mu_{n} B_{R F} \exp \left(i \omega_{0} t\right) \\
\mu_{n} B_{R F} \exp \left(-i \omega_{0} t\right) & \mu_{n} B_{0}
\end{array}\right) \Psi(t) .
$$

When $\omega_{0}$ satisfies the resonance condition Eq. (2), the solution of the Schrödinger equation for a neutron leaving the coil at time $t_{1}+\tau$ (where $\tau=l / v$ and $t_{1}$ is the time at which the neutron enters the coil) can be written [6] as

$$
\Psi\left(t_{1}+\tau\right)=\hat{C}\left(t_{1}, \tau\right) \Psi\left(t_{1}\right)
$$

where $\hat{C}\left(t_{1}, \tau\right)$ is a $2 \times 2$ matrix of the form

$$
C\left(t_{1}, \tau\right)=\left(\begin{array}{cc}
\cos (\xi) \exp \left(i \omega_{0} \tau / 2\right) & -i \sin (\xi) \exp \left(i \omega_{0}\left(t_{1}+\tau / 2\right)\right) \\
-i \sin (\xi) \exp \left[-i \omega_{0}\left(t_{1}+\tau / 2\right)\right] & \cos (\xi) \exp \left(-i \omega_{0} \tau / 2\right)
\end{array}\right)
$$

Here we introduced $\xi=\left(2 \mu_{n} / \hbar\right) B_{R F} \tau / 2$.

The spin-flip probability $\rho$ of the RF flipper plays an important role in the distribution of the neutron wave density over $2^{N}$ different waves in this many-resonance experiment. One can derive the expression for $\rho$ of the single coil from Eq. (7). We assume the initial occupation numbers $\alpha\left(t_{1}\right)$ $=1$ and $\beta\left(t_{1}\right)=0$; then the spin-flip probability is given by the occupation number of the spinor component $\downarrow$ after the coil:

$$
\rho=\beta^{*}\left(t_{1}+\tau\right) \beta\left(t_{1}+\tau\right)=\sin ^{2}(\xi) .
$$

So a distribution of the neutron wave density over the two states is described as square of sin and cos functions with the argument $\xi$. Hence $\rho$ depends on the value of the amplitude $B_{R F}$ and time $\tau$, which is proportional to the neutron wavelength $\lambda$.

Next the neutrons fly through a homogeneous magnetic field $B_{1}$ of length $L$. The effect of the magnetic field on the neutron can be expressed in a matrix language through the operator $\hat{H}$, given by

$$
H=\left(\begin{array}{cc}
\exp (i \phi) & 0 \\
0 & \exp (-i \phi)
\end{array}\right)
$$

where $\phi=-\left(\mu_{n} / \hbar\right) B_{1} T$ and $T=L / v$ is the time of flight between flippers.

Then, after $N$ resonance coils separated by $N-1$ areas with a field of strength $B_{1}$ and of length $L$ the neutron wave function has the following form:

$$
\begin{aligned}
\Psi\left(t_{1}+N \tau+(N-1) T\right)= & C\left(t_{N}, \tau\right) H C\left(t_{N-1}, \tau\right) H \cdots C\left(t_{2}, \tau\right) \\
& \times H C\left(t_{1}, \tau\right) \Psi\left(t_{1}\right),
\end{aligned}
$$

where $t_{n}=t_{1}+(n-1)(T+\tau) \quad(n=1,2, \ldots, N)$ and $H$ is given by Eq. (9). The polarization component $P_{i}$ is found by calculating $\left\langle\sigma_{i}\right\rangle=\left\langle\Psi^{*}\left(t_{1}+N \tau+(N-1) T\right)\left|\sigma_{i}\right| \Psi\left(t_{1}+N \tau\right.\right.$ $+(N-1) T)\rangle$, where $i=x, y, z$, and $\sigma_{i}$ are corresponding Pauli matrices. We derive now analytical expressions for $P_{i}$. The main difficulty here is that the operator transferring the initial wave function to the final one [Eq. (10)] is a product containing $N+1$ different matrices. We show now that the wave function Eq. (10) can be represented in a more universal form allowing one to obtain analytical expressions for the polarization components.

It can be seen from the definition of $C\left(t_{n}, \tau\right)[$ Eq. (7)] that its eigenvalues do not depend on $t_{n}$. Simple calculations give that they can be represented as $\exp (i \theta)$ and $\exp (-i \theta)$, where $\theta$ is defined as

$$
\cos \theta=\sqrt{1-\rho} \cos \left(\omega_{0} \tau / 2\right)
$$

and $\sin \theta=\sqrt{1-\cos ^{2} \theta}$ with plus before the square root. So the operator $C\left(t_{n}, \tau\right)$ can be represented in the form $C\left(t_{n}, \tau\right)=U\left(t_{n}\right) D U^{-1}\left(t_{n}\right)$, where $D$ is the diagonal matrix with the eigenvalues of $C\left(t_{n}, \tau\right)$ on the diagonal and $U\left(t_{n}\right)$ is the corresponding unitary matrix which is dependent on $t_{n}$. In these terms the wave function Eq. (10) can be rewritten in the following form:

$$
\Psi\left(t_{1}+N \tau+(N-1) T\right)=U\left(t_{N}\right) D U^{-1}\left(t_{N}\right) H U\left(t_{N-1}\right) D \cdots U^{-1}\left(t_{2}\right) H U\left(t_{1}\right) D U^{-1}\left(t_{1}\right) \Psi\left(t_{1}\right)
$$

which is a product of $N-1$ matrices' combinations of the form $U^{-1}\left(t_{n}\right) H U\left(t_{n-1}\right) D \quad(n=2,3, \ldots, N)$ embraced by curly brackets. Calculations show that such matrix combina- tions depend on $t_{n}$ and $t_{n-1}$ through the difference $t_{n}$ $-t_{n-1}=T+\tau$ only, which is the same for all $n$ and does not contain the initial moment $t_{1}$. So if we introduce a matrix 


$$
F=U^{-1}\left(t_{n}\right) H U\left(t_{n-1}\right) D,
$$

the neutron wave function Eq. (12) can be represented as

$$
\Psi\left(t_{1}+N \tau+(N-1) T\right)=U\left(t_{N}\right) D F^{N-1} U^{-1}\left(t_{1}\right) \Psi\left(t_{1}\right) .
$$

Simple but tedious calculations give that $F$, as introduced in Eq. (13), which is composed of unitary matrices, can be represented as a matrix describing a rotation in space over an angle $\gamma$ around an axis defined by a unit vector $\mathbf{n}$ in the following form [16]:

$$
F=\exp [i(\mathbf{n} \boldsymbol{\sigma}) \gamma / 2]=\cos (\gamma / 2)+i(\mathbf{n} \boldsymbol{\sigma}) \sin (\gamma / 2),
$$

where $\boldsymbol{\sigma}$ is the Pauli vector whose components are the corresponding Pauli matrices. The angle $\gamma$ in Eq. (15) appears to be given by

$$
\cos (\gamma / 2)=\sqrt{1-\rho} \cos \left(\phi-\omega_{0} T / 2\right)=\sqrt{1-\rho} \cos (\Delta \phi / 2),
$$

where $\Delta \phi=\left(\mu_{n} / 2 \hbar\right)\left(B_{1}-B_{0}\right) L / v$, which is identical to the phase quantum [Eq. (4)], and $\sin (\gamma / 2)=\sqrt{1-\cos ^{2}(\gamma / 2)}$ with plus before the square root. The components of the unit vector $\mathbf{n}$ are given by

$$
\begin{gathered}
n_{x}=-\sqrt{\rho} \frac{\sin \left(\phi-\omega_{0}(T+\tau) / 2\right)}{\tan \theta \sin (\gamma / 2)}, \\
n_{y}=-\sqrt{\rho} \frac{\sin \left(\phi-\omega_{0}(T+\tau) / 2\right)}{\sin (\gamma / 2)}, \\
n_{z}=\frac{\rho \cos \left(\phi-\omega_{0} T / 2\right) \cos \left(\omega_{0} \tau / 2\right)+\sin \left(\phi-\omega_{0} T / 2\right) \sin \left(\omega_{0} \tau / 2\right)}{\sin \theta \sin (\gamma / 2)}
\end{gathered}
$$

where $\phi$ and $\theta$ are introduced in Eqs. (9) and (11), respectively. Using the definitions of $\theta$ [Eq. (11)] and $\gamma$ [Eq. (16)], it is easily verified from Eqs. (17)-(19) that $n_{x}^{2}+n_{y}^{2}+n_{z}^{2}$ $=1$, as it should be. Strictly speaking there is a phase factor $\exp \left[-i \omega_{0}(T+\tau) / 2\right]$ before the operator exponent in the representation of $F$ [Eq. (15)] defined by Eq. (13) but it does not affect the observables and can be omitted. Thus the operator $F^{N-1}$, which appears in the wave-function representation Eq. (14), denotes a rotation of the neutron spin around an axis defined by the unit vector $\mathbf{n}$ with components given by Eqs. (17)-(19) over the angle $(N-1) \gamma$, where $\gamma$ is defined in Eq. (16).

We turn now to the neutron polarization component calculations using the wave-function representation Eq. (14). Let us assume first that the initial polarization of the neutron beam is along the $z$ direction and hence the initial occupation numbers are $\alpha\left(t_{1}\right)=1$ and $\beta\left(t_{1}\right)=0$. The final polarization components along $x, y$, and $z$ are denoted as $P_{z x}, P_{z y}$, and $P_{z z}$, respectively, where the first index refers to the initial polarization component. Calculations give that $P_{z y}$ and $P_{z x}$ contain only terms which are oscillating functions of time $t_{1}$ and hence do not contribute to the time-averaged polarization as is measured in the experiment. In contrast, in the expression for $P_{z z}$, there are no terms depending on $t_{1}$. As a result we have for polarization components in this case,

$$
\begin{gathered}
P_{z x}=P_{z y}=0, \\
P_{z z}=1-2 \rho \frac{\sin ^{2}(N \gamma / 2)}{\sin ^{2}(\gamma / 2)} .
\end{gathered}
$$

It is easy to show using the definition of $\gamma$ [Eq. (16)] that $\rho \leqslant \sin ^{2}(\gamma / 2) \leqslant 1$. So the value of $P_{z z}$ given by Eq. (21) lies in the range $[-1,1]$, as it should be. For the quantummechanical representation it is convenient to use the probability for the neutron spin to collapse into one of the two energy levels. The probability $R$ is related with the polarization $P_{z z}$ by

$$
R=\frac{1-P_{z z}}{2}=\rho \frac{\sin ^{2}(N \gamma / 2)}{\sin ^{2}(\gamma / 2)} .
$$

It is clear that for the case of only one flipper $(N=1)$ we have $R=\rho$.

Starting with the initial polarization along $x$ and $y$ directions one can find the remaining six polarization components. As a result of the calculations we have found that $P_{x z}$ and $P_{y z}$ contain only terms dependent on $t_{1}$ and are consequently equal to zero in the static experiment. $P_{x x}, P_{y y}$, $P_{x y}$, and $P_{y x}$ contains both $t_{1}$ dependent and independent terms. Meanwhile their final expressions are too cumbersome and we do not adduce them here.

The neutron beam in the above discussion is described by the plane wave $\exp \left(i k_{0} x\right) \exp (-i \omega t)$. Meanwhile the uncertainty in wave vector in present-day experiments is normally $\Delta k / k_{0} \sim 0.01$. So in practice one has to deal with a wave packet located in $k$ space at $k_{0}$ with a width of the order of $\Delta k$. This imposes a certain restriction on our consideration. As shown above the plane wave after passing through $N$ flippers is split into $2^{N}$ waves which differ from each other in phase. Similarly, if there is initially one wave packet it would 
be split finally into $2^{N}$ packets. Because waves with different spin projections on the field direction propagate with different velocities, these $2^{N}$ packets would be distributed along the $x$ direction and come to the detector at different times. The maximum distance between the packets can be roughly estimated using Eq. (3) as $m_{n} \mu_{n} B /\left(\hbar^{2} k_{0}^{2}\right) N(L+l)$, where $B \sim \max \left\{\left|B_{0}\right|,\left|B_{1}-B_{0}\right|\right\}, L$ is the distance between two neighboring flippers and $l$ is flipper length. In order for the packet splitting to be negligible this maximum distance should be much smaller than the packet length which is of the order of $1 / \Delta k$. This leads to the following condition:

$$
\frac{\Delta k}{k_{0}} \frac{m_{n} \mu_{n} B}{\hbar^{2} k_{0}} N(L+l) \ll 1 .
$$

If the inequality Eq. (23) is satisfied, the results obtained above for the plane-wave concept may be applied to the wave-packet concept too.

\section{NUMERICAL EXPERIMENT}

In order to verify the theoretical consideration previously done, a computer simulation was performed. The computation technique is based on successive multiplication of the matrices $\hat{C}$ [Eq. (7)] describing the action of the resonant coil and matrix $H$ [Eq. (9)] describing the action of the magnetic field $B_{1}$ in the space between the coils. The resultant neutron wave function after the passage through $N$ resonant coils was calculated using Eq. (10). Then we obtained the distribution of the quantum-mechanical (QM) probability $R=(1$ $\left.-P_{z z}\right) / 2$. As is seen from Eqs. (22) and (16), $R$ depends on two parameters of the system that one can vary. The first one is $\xi$, which determines the spin-flip probability of the resonant coil $\rho=\sin ^{2} \xi$ [Eq. (8)]. Here $\xi=\left(2 \mu_{n} / \hbar\right) B_{R F} l / 2 v$ where $B_{R F}$ is the amplitude of the RF field, $l$ is the length of the RF coil, and $v$ is the neutron velocity. We vary it from 0 to $2 \pi$. The other parameter is the quantum of phase $\Delta \phi$ $=\left(2 \mu_{n} / \hbar\right)\left(B_{1}-B_{0}\right) L / v$ [Eq. (4)] determined by the field $B_{1}$. Obviously, the picture of QM probability is ruled by the number of resonant coils $N$ involved in the process. This can be considered as the third parameter. It should be pointed out that the resultant curves for $R$ obtained by computational technique coincide completely with those plotted on the basis of the expression Eq. (22) which will be used in further analysis of $R$.

Figure 3 presents a two-dimensional picture of the distribution of the QM probability $R$ when the number $N$ of the resonant coils is 100 . The horizontal axis is the phase $\Delta \phi$ varied from 0 to $2 \pi$. The vertical axis is the phase $\xi$ (varied from 0 to $2 \pi$ ) of the flipping efficiency of one flipper $\rho$. We denote the picture as a "quantum carpet" in analogy with space-time pictures obtained in Ref. [6]. The dark areas correspond to a low QM probability. The lines forming the carpet are contours corresponding to the QM probability $R$ $=0.2,0.4,0.6,0.8$, respectively. As well seen from the picture, the multiwave interference creates a periodical structure with sharp and high regularity inside the period of $\xi$ and $\Delta \phi$. It should be noticed that for all $N$ the period of the function $R$

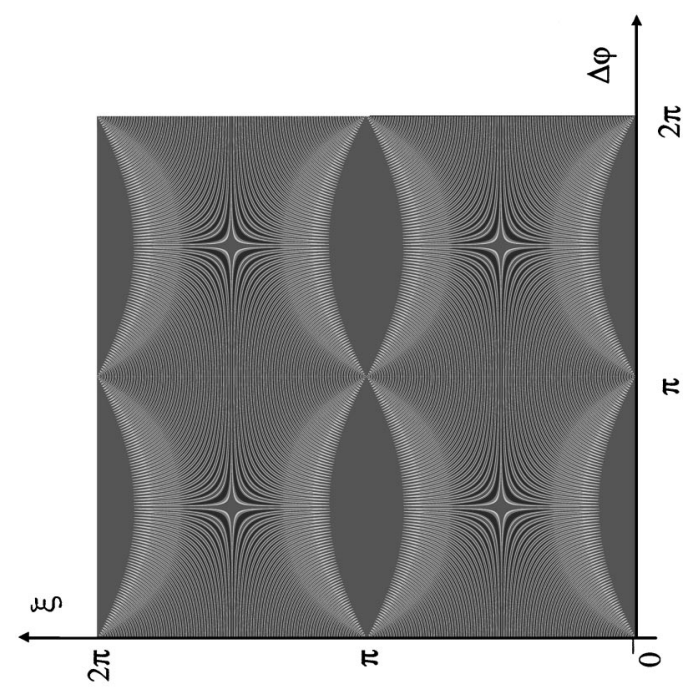

FIG. 3. "Quantum carpet," i.e., the two-dimensional picture of the distribution of the quantum-mechanical probability $R=(1$ $\left.-P_{z z}\right) / 2$ as obtained by a computer calculation based on Eq. (10) for 100 resonant coils $(N=100)$. The horizontal axis denotes the phase quantum $\Delta \varphi$ [Eq. (4)] collected in each segment with field $B_{1}$. The vertical axis denotes the phase $\xi$ (between 0 and $2 \pi$ ) of the spin-flip probability $\rho$ in every resonant coil.

in $\Delta \phi$ and in $\xi$ is $\pi$ rather than $2 \pi$. This can also be realized from Eqs. (22) and (16) and from the fact that $\cos (\gamma / 2)$ changes from $\sqrt{1-\rho}$ to $-\sqrt{1-\rho}$ as $\Delta \phi$ goes from 0 to $2 \pi$.

The increase of the number of the resonant coils leads to the appearance of sophisticated patterns with $\pi$ periodicity along the $\Delta \phi$ axis. We plot $R$ as a function of the phase $\Delta \phi$ (Fig. 4) for systems with different numbers of coils and for $\xi=\pi / 4$, i.e., spin-flip probability $\rho$ equal to $1 / 2$. According to Eqs. (22) and (16) we get $R=\cos ^{2}(\Delta \phi / 2)$ for $N=2$, i.e.,

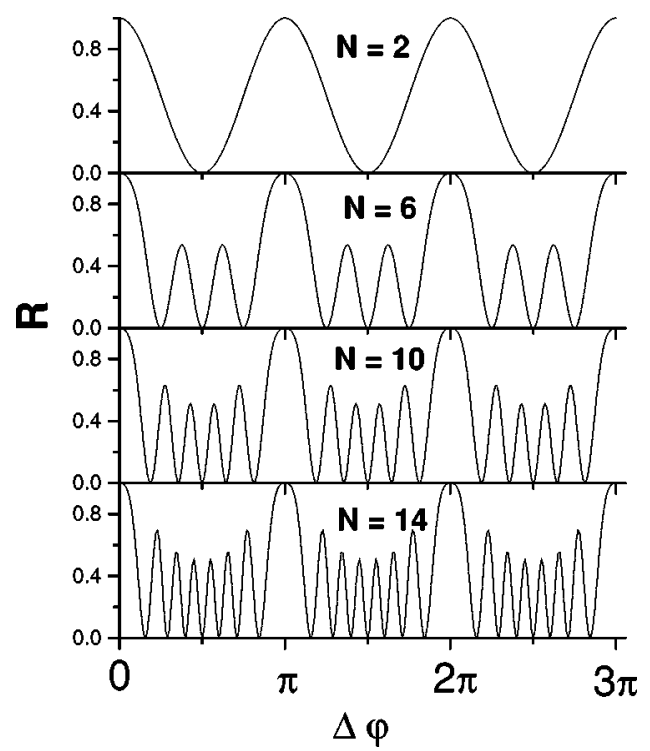

FIG. 4. Dependence of the quantum-mechanical probability $R$ on the phase quantum $\Delta \phi$ for systems of resonance coils with number of coils $N=2,6,10,14$ and for spin-flip probability $\rho$ in the resonance coils equal to $1 / 2$. 


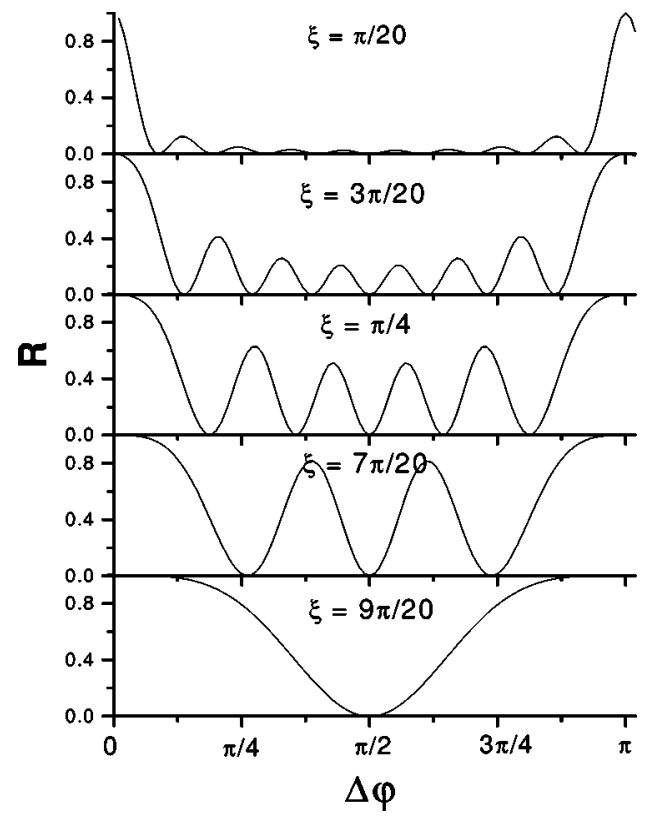

FIG. 5. Dependence of the quantum-mechanical probability $R$ for a system of ten coils $(N=10)$ on the phase quantum $\Delta \phi[\mathrm{Eq}$. (4)] for spin-flip probability $\rho$ written as $\sin ^{2} \xi$ where $\xi$ $=m \pi /(2 N)$ with $m=1,3,5,7,9$.

the $\mathrm{QM}$ probability has the cos dependence in agreement with Ramsey's calculations [6,7]. Secondary peaks appear in the pattern in the space between two main maxima. Their number $n_{\text {sec }}$ depends on the number of resonant coils $N$ and $\rho$. The number of secondary peaks is given by the simple relation $n_{s e c}=(N-2) / 2$ (see Fig. 4). It can be understood bearing in mind that $\gamma / 2$ changes from $\pi / 4$ to $\pi / 2$ as $\Delta \phi$ goes from 0 to $\pi$. Hence the number of zeros of $R$ Eq. (22) on the interval $[0, \pi]$ is $N / 2$. As seen from Fig. 4, the main maxima become narrower as the number of the coils in the system increases.

It is interesting to consider the cases when the amplitudes of the different interfering waves differ from each other. This will happen if $\rho \neq 1 / 2$. Figure 5 shows the dependence of the QM probability $R$ on the phase $\Delta \phi$ for $N=10$; the argument $\xi$ in the spin-flip probability $\rho=\sin ^{2} \xi$ was taken equal to $m \pi /(2 N)$ with $m=1,3,5,7,9$. For these values of $\xi$ the QM probability has maxima at $\Delta \phi=n \pi$, i.e., it is periodically self-reconstructing. The number of secondary maxima between the main ones decreases but their height increases as $\xi$ grows by increasing $m$.

It is also of interest how the QM probability behaves at constant $\Delta \phi$. Figure 6 shows the QM probability $R$ as a function of the phase $\xi$ for $N=10$ and for the value of the phase quantum $\Delta \phi=k \pi / N$ with $k=0,1,2,3,4$. As is clear from Eq. (16), in the case of $\Delta \phi=0$ (top picture) $\gamma / 2=\xi$ and $R=\sin ^{2}(N \xi)$. So ten maxima appear, in accordance with the number $N$ of resonant coils. The number of maxima decreases as $\Delta \phi$ increases from 0 to $\pi / 2$. Two peaks per step $\Delta \phi=\pi / N$ vanish. For $\Delta \phi=\pi / 2$ the QM probability $R$ becomes equal to 0 . A further increase of the phase quantum $\Delta \phi$ results in a full reconstruction of the pattern at $\Delta \phi$ $=\pi$.

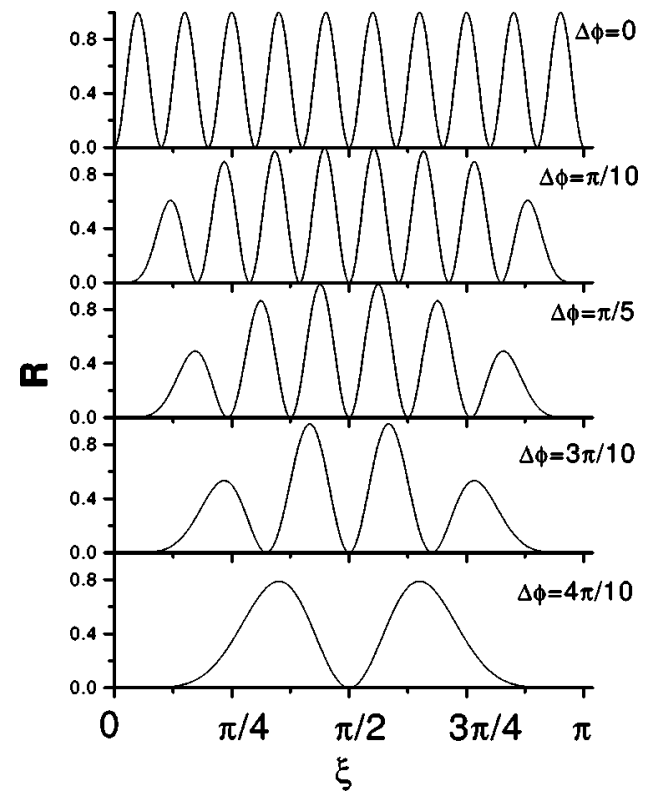

FIG. 6. Dependence of the quantum-mechanical probability $R$ for a system of ten coils $(N=10)$ on the argument $\xi$ in the spin-flip probability $\rho$ written as $\sin ^{2} \xi$ when the phase quantum $\Delta \phi$ $=k \pi / N$ with $k=0,1,2,3,4$.

\section{ACTUAL EXPERIMENT}

The measurements described in this paper were carried out at the reactor WWR-M in St. Petersburg Nuclear Physics Institute. The outline of the experiment is shown in Fig. 7. A system consisting of six RF coils and five small dc coils is set in the space between two large dc coils producing the static field $B_{0}$. We set $B_{0}$ equal to $30 \mathrm{G}$ and the frequency $\omega_{0}$ of the oscillating field equal to $89 \mathrm{kHz}$ to fulfill the resonance condition Eq. (2). The length $l$ of the RF coils is $0.04 \mathrm{~m}$ and they are placed at a distance $L$ of $0.02 \mathrm{~m}$ from each other. The small dc coils with thickness of $0.02 \mathrm{~m}$ produce a static

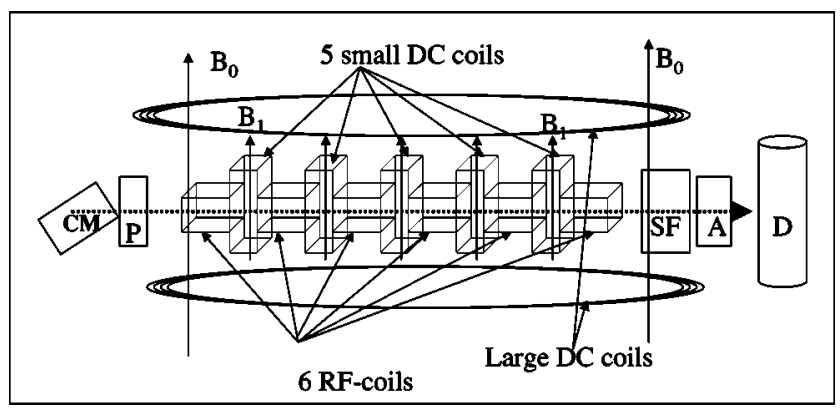

FIG. 7. Schematic drawing of the experiment: MC, monochromator crystal; $P$, polarizer; SF, spin flipper; $A$ analyzer; and $D$, detector. The system consisting of six resonance coils [radio frequency (RF) spin flippers] and five small dc coils SC is located between the polarizer $P$ and the analyzer $A$ in the magnetic field $B_{0}$ produced by a pair of large dc coils. The quantum-mechanical probability $R$ was measured as a function of the field $\Delta B$ simultaneously generated in the five small dc coils (which determine the phase quantum $\Delta \phi$ ) and as a function of $B_{R F}$ simultaneously generated in the six RF coils (which determine the argument $\xi$ of the spin-flip probability $\left.\rho=\sin ^{2} \xi\right)$. 

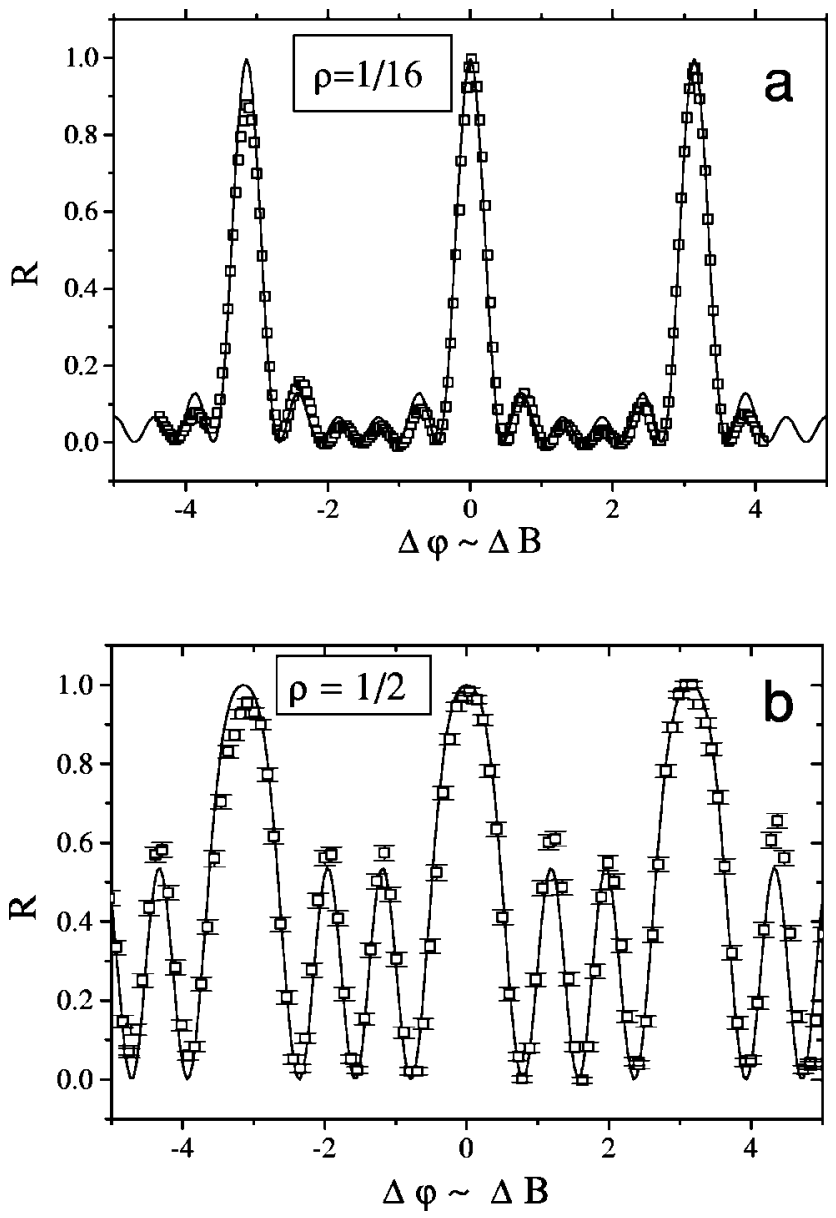

FIG. 8. Dependence of the quantum-mechanical probability $R$ for a system of $N=6$ coils on the phase quantum $\Delta \phi \sim \Delta B$ and at the spin-flip probability $\rho=1 / 16$ (a) and $1 / 2$ (b).

magnetic field $\Delta B$ parallel to the main static magnetic field $B_{0}$. Thus the superposition of the fields of the large dc coils and the small dc coils make the resultant dc field between RF coils equal to $B_{1}=B_{0}+\Delta B$. A neutron beam polarized along the magnetic field $B_{0}\left(P_{0}=0.95\right)$ and monochromatized at $\lambda=0.23 \mathrm{~nm}(\Delta \lambda / \lambda=0.02)$ enters the system of coils. The polarization component along the field is analyzed by the spin flipper (SF) and the analyzer $(A)$.

The spin-flip probability $\rho$ was set by adjusting the amplitude of $B_{R F}$ in all six RF coils simultaneously such that the phase $\xi=\left(2 \mu_{n} / \hbar\right) B_{R F} l / 2 v=m \pi / 2 N$, where the number of the resonance coils $N=6$. The polarization was measured as a function of the magnetic field $\Delta B$ produced by all small dc coils simultaneously. In fact the phase quantum $\Delta \phi$ $=\left(2 \mu_{n} / \hbar\right) \Delta B L / v$ was varied in this way. The $\mathrm{QM}$ probability $R$ was calculated from the polarization $P_{z z}$ using Eq. (22).

Figure 8 shows the measured value of the probability $R$ as a function of the phase quantum $\Delta \phi$ for $\rho=1 / 16$ (a) and $1 / 2$ (b), set as $\sin ^{2} \xi$ where $\xi=\pi / 12$ and $\xi=\pi / 4$, respectively. The symbols represent the experimental data and the lines correspond to the theoretical predictions according to Eqs. (16) and (22). In spite of some imperfection of the experimental picture, the theoretical curves match well the experi- mental points. Thus, the situation of the multiple splitting of a neutron wave is experimentally realized using the neutron resonance technique.

\section{CONCLUDING REMARKS}

In this paper we give a theoretical description of polarized neutron multiresonance experiments. The description shows that the system of $N$ resonant coils produces a large number of neutron waves. These waves interfere and each pair of them contributes to highly regular patterns in quantummechanical probability. We have introduced the spin-flip probability $\rho$ and the field $B_{1}$ between coils as key parameters of this system. The analytical expression for QM probability was obtained for arbitrary values of $\rho$ and $B_{1}$. This expression was testified by the computer calculations and by the experiment. The experimental data are well described by the proposed theoretical picture.

This experiment has an analogy with an experiment in optics: light diffraction in a grating [1,2]. Our experiment as well as those made in Refs. [8-15] may be referred to as the field of neutron resonance interferometry (NRI). A certain difference between NRI and optics is discussed in Refs. [1012]. Thus, the interference pattern in NRI appears in phase space while the analogous pattern in optical devices appears in real space. In spite of the difference, the obtained analytical expression [Eq. (22): $\left.R \sim \sin ^{2}(N \gamma / 2) / \sin ^{2},(\gamma / 2)\right]$ shows the analogy with optics in a most obvious way. The interference pattern in the NRI experiment is the resultant of many waves with different phases but with the same energy as it may be observed in a plane behind a diffraction grating of $N$ slits. To see better the similarity and the difference between $\mathrm{N}$-resonance and $\mathrm{N}$-slit experiments, we may refer to the paper by Ramsey [7], where he discussed the problem of the complementarity in neutron two-path interference and tworesonance-field interference.

The discussed experiment is also obviously linked with nuclear magnetic resonance studies, which nowadays routinely involve sequences of RF pulses to manipulate nuclear spins. In turn, nuclear-magnetic-resonance (NMR) spectroscopy is proposed as a basis for quantum computation [17]. Without going deep into the problem we emphasize only that the experiment discussed in the present paper allows one to manipulate the quantum state of a neutron in full analogy with the NMR technique.

It is also peculiar in this experiment that the neutron as a Gaussian wave packet propagates through the system of $N$ $>2$ resonant coils and therefore splits into $N>2$ wave packets. When one uses this system for a scattering experiment in a way similar to the neutron spin echo, then the correlation function obtained consists of many-point contributions and is different from the pair-correlation function, which is a standard object for the study.

Therefore we conclude that the problem of multiwave interference may be of interest both from theoretical and experimental point of view. 


\section{ACKNOWLEDGMENTS}

The authors would like to thank L.A. Axelrod for his help in performing the experiment. This work was part of the research program of the "Stichting voor Fundamenteel Onderzoek der Materie (FOM)," which is financially sup- ported by the "Nederlandse Organisatie voor Wetenschappelijk Onderzoek (NWO)." The authors thank RFFR (Projects Nos. SS-1671.2003.2 and 03-02-17340) and the Russian State Programs "Neutron Research of the Condensed State" and "Quantum Macrophysics."
[1] M.V. Berry and S. Klein, J. Mod. Opt. 43, 2139 (1996).

[2] M.V. Berry and E. Bodenschatz, J. Mod. Opt. 46, 349 (1999).

[3] A.E. Kaplan, I. Marzoli, W.E. Lamb, Jr., and W.P. Schleich, Phys. Rev. A 61, 032101 (2000).

[4] C. Leichtle, I.Sh. Averbukh, and W.P. Schleich, Phys. Rev. A 54, 5299 (1996).

[5] J.H. Eberly, N.B. Narozhny, and J.J. Sanchez-Mondragon, Phys. Rev. Lett. 44, 1323 (1980).

[6] N.F. Ramsey, Molecular Beams (Oxford University Press, Oxford, 1990).

[7] N.F. Ramsey, Phys. Rev. A 48, 80 (1993).

[8] S.V. Grigoriev, W.H. Kraan, F.M. Mulder, and M.Th. Rekveldt, Phys. Rev. A 62, 63601 (2000).

[9] F.M. Mulder, S.V. Grigoriev, W.H. Kraan, and M.Th. Rekveldt,
Europhys. Lett. 51, 13 (2000).

[10] R. Golub and R. Gähler, Phys. Lett. A 123, 43 (1987).

[11] R. Gähler and R. Golub, J. Phys. (Paris) 49, 1195 (1988).

[12] R. Golub, R. Gähler, and T. Keller, Am. J. Phys. 62, 779 (1994).

[13] G.M. Drabkin and R.A. Zhitnikov, Sov. Phys. JETP 11, 729 (1960).

[14] B. Alefeld, G. Badurek, and H. Rauch, Z. Phys. B: Condens. Matter 41, 231 (1981).

[15] G. Badurek, H. Rauch, and J. Summhammer, Phys. Rev. Lett. 51, 1015 (1983).

[16] L.D. Landau and E.M. Livshitz, Quantum Mechanics (Pergamon, Oxford, 1977).

[17] N.A. Gershenfeld and I.L. Chuang, Science 275, 350 (1997). 